\title{
Substrates for Zero Temperature Coefficient Love Wave Sensors
}

\author{
Bernhard Jakoby, Senior Member, IEEE, and Michael J. Vellekoop
}

\begin{abstract}
Microacoustic Love wave delay lines show high sensitivity to perturbations such as mass depositions on the wave-guide surface. Furthermore, because of their shear polarization, Love waves are ideally suited for liquid sensing applications. Using a Love wave delay line as feedback element in an oscillator allows the realization of viscosity sensors, and, using a chemical interface, chemical sensors, where the output signal is the oscillation frequency. To achieve a high effective sensitivity, the cross-sensitivity to temperature has to be kept low. We outline the proper choice of a material and especially focus on the influence of crystal cut and the major device design parameters (mass sensitivity and coupling coefficient) on the temperature coefficient of the sensor.
\end{abstract}

\section{INTRODUCTION}

$\mathrm{A}$ SIMPLE Love mode waveguide consists of a guiding layer deposited on a substrate [Fig. 1(a)]. Generally, shear polarized waves do not couple with compressional modes in an adjacent liquid so that corresponding acoustic radiation losses are avoided. Apart from the advantageous shear polarization, Love waves show a high sensitivity with respect to surface mass loading thanks to the effective guidance of the wave close to the sensing surface. This guidance is achieved through the thin guiding layer that is deposited on a (piezoelectric) substrate [Fig. 1(a)]. For a summary of the advantageous properties of Love wave delay lines for liquid sensing applications, see [1]-[3].

Incorporating a Love wave delay line as a feedback element in an oscillator [Fig. 1(b)], a surface mass loading can be sensed with high resolution and sensitivity by monitoring the oscillator frequency. Loading the device with viscous liquids, a liquid layer becomes entrained with the shear movement at the surface, which leads to an effective surface mass loading. This effect can be used for viscosity sensing [4]. Using a selectively adsorbing chemical interface layer, a chemical sensor for liquid phase applications can be realized. In this contribution, we focus on "bare" Love wave devices as they are used for viscosity sensing; however, the described method can be extended mutatis mutandis to devices featuring selective overlayers for chemical sensing.

Manuscript received February 22, 1999; accepted November 23, 1999. This work was supported by the Brite-Euram project BE-951745: MIMICS.

The authors are with Delft University of Technology, Electronic Instrumentation Lab./DIMES, Mekelweg 4, 2628 CD Delft, The Netherlands (e-mail: Bernhard.Jakoby@bosch.com). Present address of Bernhard Jakoby: Robert Bosch GmbH, Sensor Technology Center K8/SPP5, Tübinger Strasse 123, D-72762 Reutlingen, Germany.
To achieve a high effective sensitivity ${ }^{1}$, the cross sensitivity to temperature has to be kept low. Typically, a $85 \mathrm{MHz}$ oscillator using a Love wave delay line on ST-cut quartz has a temperature sensitivity of about $2.5 \mathrm{kHz} / \mathrm{K}$. The short-term stability (frequency noise) of the oscillator is typically a few hertz. This noise prescribes the ultimate detection limit for the sensor if the frequency changes caused by device temperature changes can be kept below the frequency noise. For the specified temperature coefficient, this would mean that the device temperature would have to be kept within about $0.001 \mathrm{~K}$. This temperature dependence can be compensated to a certain extent by using a reference setup with two oscillators in which only one contains a sensing delay line. Taking the difference, frequency compensates the temperature dependence of the device. Practically, such a reference setup can reduce the temperature dependence by a factor of ten or higher. But, to achieve optimum temperature stability, we aim for a small or zero temperature coefficient of the sensing device, avoiding the need for a temperature control. This can be achieved by choosing appropriate materials, crystal cuts, and design parameters for the Love wave device. In this paper, we discuss analysis results and confirm experimental results, which help to find a reasonable trade-off in the design of a sensor.

\section{THEORY}

The oscillation condition of the oscillator is given by

$$
\beta L+\phi_{t}+\phi_{a}=2 \pi n
$$

where $\beta=2 \pi / \lambda$, denotes the wavenumber of the propagating mode ( $\lambda=$ wavelength); $L$, is the effective length of the delay line; $\phi_{t}$, and $\phi_{a}$, are the phase shifts introduced by the interdigital transducers (IDTs) and the amplifier, respectively; and $n$, is an integer (mode number). The quantities $\beta$ and $\phi_{t}$ depend on the frequency $f$, as well as the device temperature $T ; L$ depends only on $T$; and $\phi_{a}$ depends only on $f^{2}$.

To determine the dependence of the frequency on the device temperature $d f / d T$, we consider the complete

\footnotetext{
${ }^{1}$ Using the term "effective sensitivity," we refer to the fact that reliable measurements can only be made if noise and drift effects are kept small. The latter determines the sensitivity that can effectively be used as expressed, e.g., by the detection limit.

${ }^{2}$ We do not consider the influence of the amplifier temperature drift, i.e., we assume the amplifier to be at a constant temperature.
} 
derivative of (1) with respect to $T$, and divide the resulting equation by $\beta L$, yielding

$$
\frac{1}{\beta} \frac{\partial \beta}{\partial f} \frac{d f}{d T}+\frac{1}{\beta} \frac{\partial \beta}{\partial T}+\frac{1}{L} \frac{\partial L}{\partial T}+\frac{1}{\beta L} \frac{\partial\left(\phi_{t}+\phi_{a}\right)}{\partial f} \frac{d f}{d T}+\frac{1}{\beta L} \frac{\partial \phi_{t}}{\partial T}=0
$$

Especially for long delay lines $(\beta L \gg 2 \pi)$, the last two terms can be neglected (see [5] for an evaluation of orders of magnitude), such that, after some algebraic manipulations, we finally obtain the temperature coefficient of the oscillation frequency (TCF):

$$
\mathrm{TCF}=\frac{1}{f} \frac{d f}{d T}=\frac{v_{g}}{v} \underbrace{\left(\frac{1}{v} \frac{\partial v}{\partial T}-\frac{1}{L} \frac{\partial L}{\partial T}\right)}_{-\mathrm{TCD}} .
$$

Here, we expressed the result in terms of $v=2 \pi f / \beta$, and $v_{g}=2 \pi(\partial \beta / \partial f)^{-1}$, the phase and group velocities of the wave, respectively. The bracketed expression on the righthand side is the negative temperature coefficient of delay (TCD). Because the Love wave is dispersive $\left(v_{g} \neq v\right)$, the TCF and -TCD are not equal but a correction factor $v_{g} / v$, has to be considered.

Apart from a small or zero temperature coefficient, we aim for a high coupling coefficient $\kappa^{2}$, and a high sensitivity. The sensitivity can be described in terms of the relative frequency change caused by an increase in the surface mass loading $\rho_{s}$ :

$$
S_{f}=\frac{1}{f} \frac{d f}{d \rho_{s}}=\frac{v_{g}}{v} S_{v}=\frac{v_{g}}{v} \frac{1}{v} \frac{\partial v}{\partial \rho_{s}} .
$$

This equation gives the relationship of $S_{f}$, to the relative sensitivity of the phase velocity $S_{v}$, which again contains the dispersion-related correction factor $v_{g} / v[3]$.

\section{ANALYSIS}

The TCF, $\kappa^{2}$, and $S_{f}$, have been numerically analyzed for a Love mode waveguide by using the propagator matrix approach [3], [6]. The material parameters and their temperature dependence ${ }^{3}$ have been taken from [7]-[9]. The analysis has been performed for $25^{\circ} \mathrm{C}$; for temperature coefficients, the material parameters at $35^{\circ} \mathrm{C}$ have also been used to approximate temperature coefficients by means of difference quotients. For the thermal extension, it has been assumed that the expansion coefficient of the substrate material is essentially valid also for the coated substrate [9].

For Rayleigh surface acoustic waves (SAWs), corresponding investigations have been started almost three decades ago. One of the major results was the discovery of the ST-cut for quartz that yields a zero TCD, for the Rayleigh SAW [10]. For other materials such as $\mathrm{LiTaO}_{3}$,

\footnotetext{
${ }^{3}$ For quartz, temperature coefficients for permittivity are not available but can be neglected because of the comparatively weak piezoelectric effect in quartz.
}

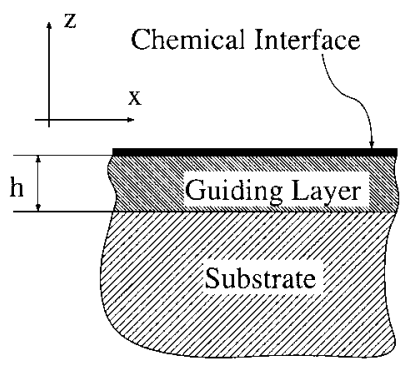

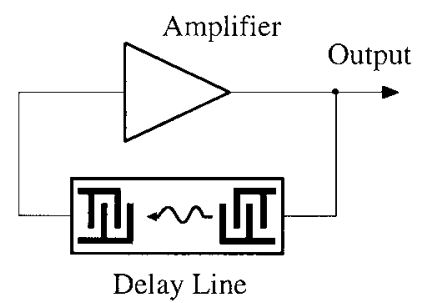

b.
Fig. 1. a) Cross section of waveguide; b) oscillator setup.

efforts have been made to achieve zero TCD, by adding an overlayer (e.g., $\mathrm{SiO}_{2}$ ), the thickness of which allows tuning of the TCD [9]. This configuration yields a dispersive generalized Rayleigh SAW. Apart from the different polarization of the utilized SAW, the problem is similar to the Love mode waveguide investigated here. In fact, the polarization is a severe restriction in our search for the optimum configuration because we want to obtain a pure (shear) polarization of the wave in any case. Otherwise, the aforementioned coupling to compressional waves in the adjacent liquid would occur, which immediately leads to a serious damping of the wave. As a consequence, the piezoelectric substrate must be oriented such that electric field components generated by the IDTs couple only to shear movements of the crystal. Let us consider resulting conditions for the material parameter matrices in abbreviated notation [11] corresponding to the constitutive equations

$$
\begin{aligned}
& \mathbf{T}=\underline{\underline{c}} \cdot \mathbf{S}-\underline{\underline{e}}^{T} \cdot \mathbf{E}, \\
& \mathbf{D}=\underline{\underline{e}} \cdot \mathbf{S}+\underline{\underline{\epsilon}} \cdot \mathbf{E} .
\end{aligned}
$$

The matrices $\underline{\underline{c}}, \underline{\underline{e}}$, and $\underline{\underline{\epsilon}}$, represent the elastic, piezoelectric, and permittivity tensors, respectively. The vectors $\mathbf{D}, \mathbf{E}, \mathbf{T}$, and $\mathbf{S}$, represent the dielectric displacement, the electric field, and the elastic stresses and strains, respectively. We assume wave propagation in the $x$, direction [see co-ordinate system in Fig. 1(a)] and adopt a 2-D approximation assuming $\partial / \partial y=0$, within the Love wave beam excited by the IDTs. If the material matrices $\underline{\underline{c}}$ and $\underline{\underline{e}}$ obey the following structures ${ }^{4}$

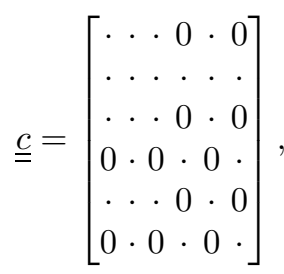

$$
\begin{aligned}
& \underline{\underline{e}}=\left[\begin{array}{c}
0 \cdot 0 \cdot 0 \cdot \\
\cdots \\
0 \cdot 0 \cdot 0 \cdot
\end{array}\right],
\end{aligned}
$$

then the field equations decouple into equations for two sets of variables $\Sigma_{1}=\left\{u_{2}, \phi, T_{4}, T_{6}, D_{1}, D_{3}\right\}$ and $\Sigma_{2}=$

\footnotetext{
${ }^{4}$ Here the dots $(\cdot)$ denote an arbitrary entry; the zeros indicate necessary zero entries.
} 
$\left\{u_{1}, u_{3}, T_{1}, T_{3}, T_{5}\right\}$. The IDTs associated with an $x$, propagating wave predominantly (neglecting finger end fields) generate electric field components in the $x$ - and $z$ - direction such that only coupling with shear movements corresponding to set $\Sigma_{1}$, is obtained. Note that this does not mean that no generalized Rayleigh mode can propagate in the structure for example. It only means that the electric field components generated by the IDTs do not excite such modes. If all materials in the considered structure (for isotropic, non-piezoelectric media, these conditions are trivial) fulfill these conditions, the pure shear polarization of excited wave is maintained because none of the boundary conditions introduced by material interfaces introduces coupling between the field components of the sets $\Sigma_{1}$ and $\Sigma_{2}[3]$.

The conditions (7) turn out to be very stringent requirements for common substrate materials. When testing different crystal orientations defined by Euler angles $(\lambda, \mu, \theta),[7], \mathrm{LiNbO}_{3}$, as well as $\mathrm{LiTaO}_{3}$, did not show any promising regions in the 3 -D $\lambda, \mu, \theta$,-space where conditions (7) were met exactly. In quartz, however, the line $\left(0^{\circ}, \mu, 90^{\circ}\right)$, proved to support pure shear mode excitation. Note that the common ST-cut is a point on this line at $\mu=132.75^{\circ}$. For Rayleigh wave propagation on ST-cut quartz wafers, $\theta=0^{\circ}$, is utilized, and pure shear mode propagation requires $\theta=90^{\circ}$. Actually, the Love wave devices fabricated at our lab so far utilized standard ST-cut quartz with propagation direction $\theta=90^{\circ}$. As the guiding layer, $\mathrm{SiO}_{2}$, has been considered, where material data for fused quartz have been used in the analysis. For the devices we fabricated, plasma-enhanced chemical vapor deposition (PECVD) has been used to deposit this layer, which is known to obey somewhat different but not accurately known material parameters (see subsequent text).

For the sake of completeness, we note that also $\mathrm{LiNbO}_{3}$, and $\mathrm{LiTaO}_{3}$, obey cuts, which allow the excitation of almost shear polarized waves, which can be sufficient for a number of applications. Recently, new quartz-like materials such as Langasite have been introduced for microacoustic devices, which also obey cuts supporting purely (or almost purely) shear polarized waves. For these materials, reliable material data (especially temperature coefficients) have yet to be determined.

\section{Results}

Apart from the crystal orientations (represented by the Euler angle $\mu$ ), the thickness of the guiding layer $h$, [Fig. 1(a)] represents a second parameter that can be varied. The TCF, the coupling factor $\kappa^{2}$, and the scaled sensitivity $S_{f} \lambda$, all depend on the scaled layer thickness $h / \lambda[3]$. Hence, we calculated these quantities a as function of $h / \lambda$, and the Euler angle $\mu$. This scaling is near at hand if one wants to optimize for TCF, coupling and sensitivity for a prescribed wavelength $\lambda$. As the latter is intimately connected to the IDT periodicity, an optimum for a given IDT finger spacing can be obtained. It turns out that not the en-

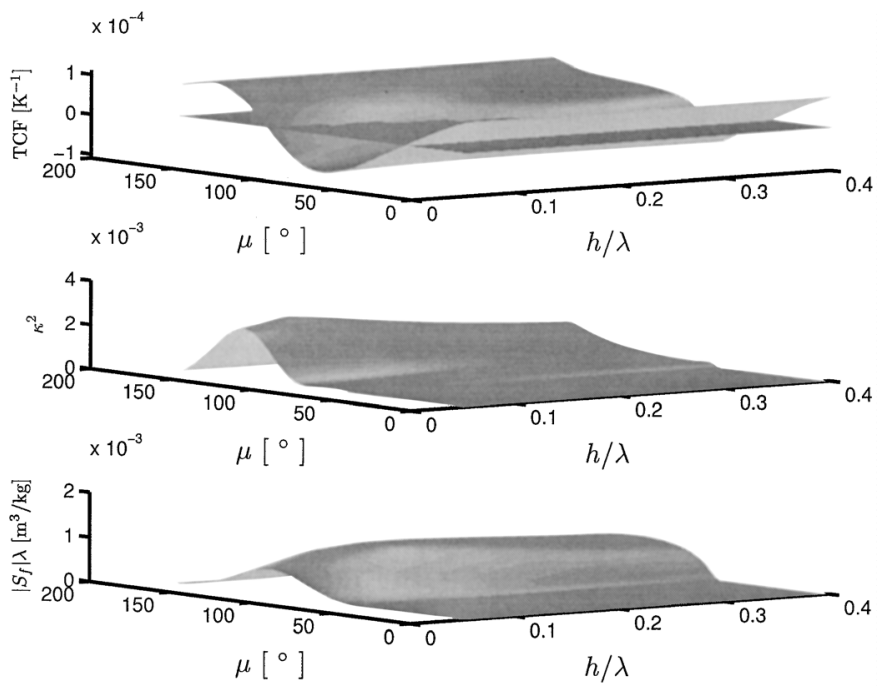

Fig. 2. TCF (cut by $T C F=0$ plane), $\kappa^{2}$, and $\left|S_{f}\right| \lambda$, as functions of $\mu$, and $h / \lambda$. The other Euler angles are $\lambda=0$, and $\theta=90^{\circ}$.

tire $\mu$, range yields a guided Love wave. Only in the range of about $\mu=70^{\circ} \ldots 175^{\circ}$ a guided shear mode is obtained. Outside this range, it degenerates into a leaky mode because of the presence of bulk shear modes in the substrate that show lower velocities than those in the guiding layer, which violates the guidance condition.

Fig. 3 shows the TCF, the coupling coefficient $\kappa^{2}$, and the scaled sensitivity $\left|S_{f}\right| \lambda$, as functions of the Euler angle $\mu$, and the relative thickness of the guiding layer $h / \lambda$. TCF is zero along a characteristic line (the cut with the zero surface in the plot). Note that the range $\mu<70^{\circ}$, corresponds to leaky modes such that the zero TCF, in this range is irrelevant. The coupling and the sensitivity show maxima around $\mu=145^{\circ}, h / \lambda=0.12$, and $\mu=125^{\circ}, h / \lambda=0.2$, respectively. This can also be seen in the combined contour plot in Fig. 3. Here, for $\kappa^{2}$ and $\left|S_{f}\right| \lambda$, contour lines at 25,50 , and $75 \%$ of their maximum values are drawn. For the TCF, the loci TCF $=0$ as well as TCF $= \pm 50 \mathrm{ppm}$ are shown. This plot suggests that at about $\mu=122^{\circ}, h / \lambda=0.1$, a zero TCF, can be achieved with $\kappa^{2}>50 \%$, and $\left|S_{f}\right| \lambda>75 \%$, of their maximum value.

For small $h / \lambda$, the substrate is becoming the material that dictates the temperature coefficient. In the limit $h / \lambda \rightarrow 0$, the zero TCF contour approaches the value $\mu=125.25^{\circ}$, corresponding to the known AT cut of quartz, which is known to yield a zero temperature coefficient for bulk shear resonators. However, small thicknesses $h / \lambda$, also yield small sensitivities and coupling coefficients. For another commercially available cut, namely the STcut $\mu=132.5^{\circ}$, higher coupling and sensitivity can be achieved, but no zero TCF, can be expected for any value of $h / \lambda$. Fig. 4 shows the TCF, vs $h / \lambda$, for Love waves on ST-cut wafers. From previous simulations, we know that the used material data for the fused quartz model the behavior of the actually deposited PECVD $\mathrm{SiO}_{2}$, layer only to a limited extent [12]. 


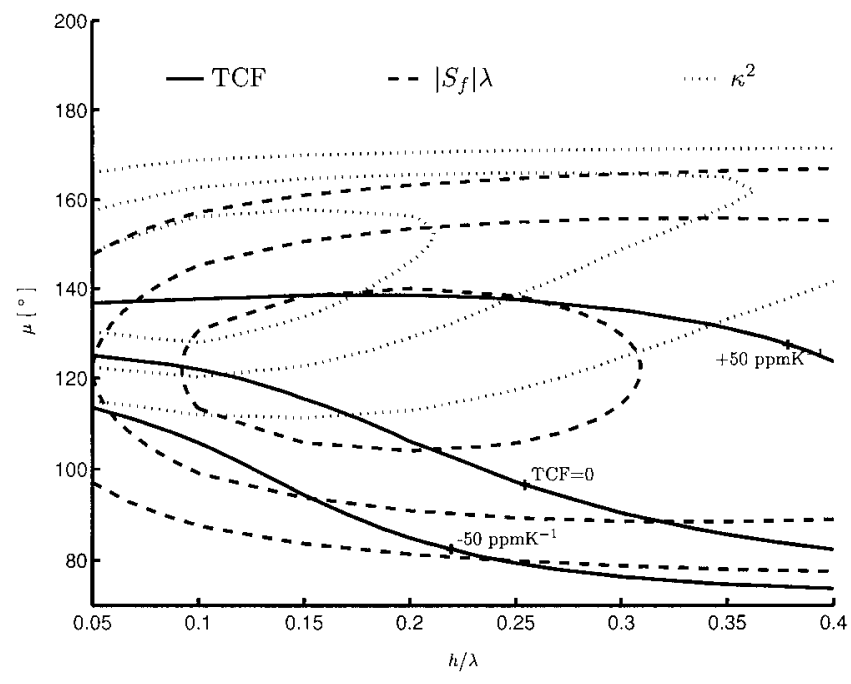

Fig. 3. Contour plot corresponding to Fig. 2. For the sake of clarity, only the contour- ines indicating 25,50 , and $75 \%$ of the maximum values of $\kappa^{2}$ and $\left|S_{f}\right| \lambda$ are shown.

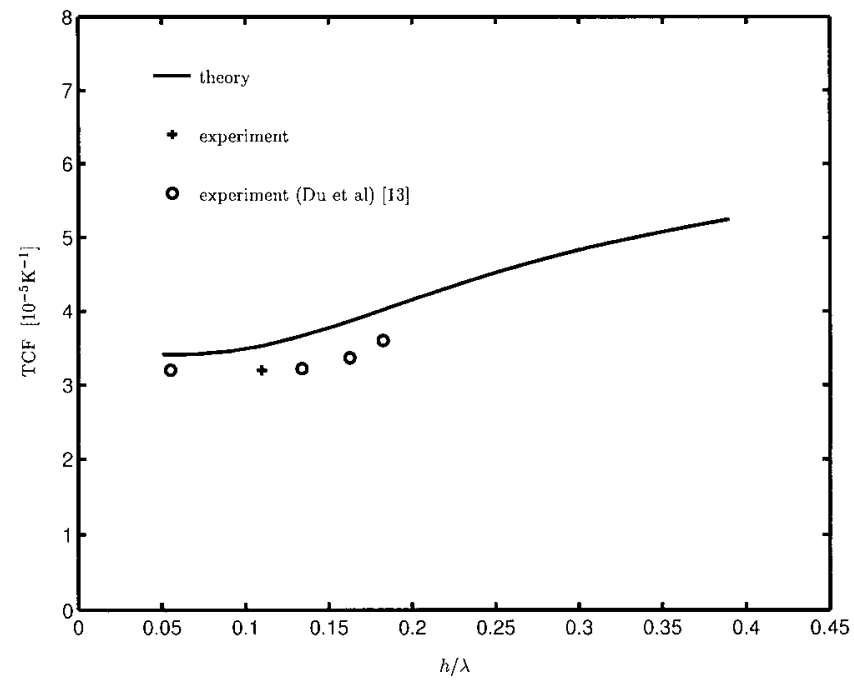

Fig. 4. Measured and calculated TCF, for varying layer thicknesses on ST-cut quartz substrate $\left(\mu=132.5^{\circ}\right)$.

To get an idea about the validity of our analysis for actually fabricated $\mathrm{SiO}_{2}$ layers, we show, in Fig. 4, measured temperature coefficients from [13] and from an experiment we conducted ourselves. The deviation from the theory is in the order of $10 \%$. The results confirm that the analysis can be used to identify optimum TCF regions and to obtain a reasonable idea about the magnitude of TCF. If a new cut around $\mu=120^{\circ}$, were available, tuning of the thickness $h / \lambda$, should allow a zero TCF. The use of other guiding layer materials is another option to achieve zero TCF. In [14], experiments with additional polymethylmetacrylate (PMMA) overlayers have been made, which allowed the researchers to obtain zero TCFs even for STcut quartz substrates. However, the use of polymers like PMMA as guiding layer, shows disadvantages in terms of acoustic damping [3]. Similar effects can be expected from chemical interface layers as soon as they exceed a certain thickness.

Further experimental results confirming these theoretical results have been obtained recently and will be reported elsewhere [15].

\section{Conclusions}

Love waves are attractive for the realization of highly sensitive microacoustic sensors. To actually obtain a high effective sensitivity, the cross sensitivity to temperature should be minimized. We analyzed the choice of optimum crystal cut and guiding layer thickness to allow a reasonable trade-off among zero temperature coefficient, high sensitivity, and high electromechanical coupling. The analysis results show that this should be achievable with a quartz substrate rotated by Euler-angles $\left(0,120\right.$, and $\left.90^{\circ}\right)$ and an $\mathrm{SiO}_{2}$ guiding layer, where the desired properties can be tuned by varying the guiding layer thickness $h$. For the further analysis of Love wave guiding structures, we note that the accurate determination of the relevant thin layer material parameters (especially the Lamé constant $\mu$ ) for the used layer materials is highly desirable.

\section{REFERENCES}

[1] G. Kovacs, G. W. Lubking, M. J. Vellekoop, and A. Venema, "Love waves for (bio)chemical sensing in liquids," in Proc. IEEE Ultrason. Symp., Tucson, AZ, pp. 281-285, 1992.

[2] E. Gizeli, A. C. Stevenson, N. J. Goddard, and C. R. Lowe, "A novel Love-plate acoustic sensor utilising polymer overlayers," IEEE Trans. Ultrason., Ferroelect., Freq. Contr., vol. 39, no. 5, pp. 657-659, 1992.

[3] B. Jakoby and M. J. Vellekoop, "Properties of Love waves: Applications in sensors," Smart Mater. Struct., vol. 6, pp. 668-679, 1997.

[4] - "Viscosity sensing using a Love wave device," Sens. Actuators $A$, vol. 68, pp. 275-281, 1998.

[5] J. D. Maines, E.G.S. Paige, A. F. Saunders, and A. S. Young, "Simple technique for the accurate determination of delay-time variations in acoustic-surface-wave structures," Electron. Lett., vol. 5, pp. 678-680, Dec. 1969.

[6] A. H. Fahmy and E. L. Adler, "Propagation of acoustic surface waves in multilayers: A matrix description," Appl. Phys. Lett., vol. 22, pp. 495-497, May 1973.

[7] A. J. Slobodnik, Jr., E. D. Conway, and R. T. Delmonico, "Microwave acoustics handbook," Air Force Cambridge Research Laboratories, Bedford, MA, Tech. Rep. AFCRL-TR-73-0597, 1973.

[8] A. J. Slobodnik, Jr., "The temperature coefficients of acoustic surface wave velocity and delay on lithium niobate, lithium tantalate, quartz, and tellurium dioxide," Air Force Cambridge Research Laboratories, Bedford, MA, Tech. Rep. AFCRL-TR72-0082, 1971.

[9] T. E. Parker and H. Wichansky, "Temperature-compensated surface-acoustic-wave devices with $\mathrm{SiO}_{2}$ film overlays," J. Appl. Phys., vol. 50, pp. 1360-1369, Mar. 1979.

[10] M. B. Schulz, B. J. Matsinger, and M. G. Holland, "Temperature dependence of surface acoustic wave velocity on $\alpha$ quartz," $J$. Appl. Phys., vol. 41, pp. 2755-2765, Jun. 1970.

[11] B. A. Auld, Acoustic Fields in Solids. vol. I, New York: John Wiley \& Sons, 1973.

[12] B. Jakoby and M. J. Vellekoop, "Analysis and optimization of Love wave liquid sensors," IEEE Trans. Ultrason., Ferroelect., Freq. Contr., vol. 45, pp. 1293-1302, Sep. 1998. 
[13] J. Du, G. L. Harding, J. A. Ogilvy, P. R. Dencher, and M. Lake, "A study of Love-wave acoustic sensors," Sens. Actuators A, vol. 56, pp. 211-219, 1996.

[14] J. Du and G. L. Harding, "A multilayer structure for Love-mode acoustic sensors," Sens. Actuators A, vol. 65, pp. 152-159, 1998.

[15] B. Jakoby, J. Bastemeijer, and M. J. Vellekoop, "Temperaturecompensated Love-wave sensors on quartz substrates," Sens. Actuators $A$, to be published.

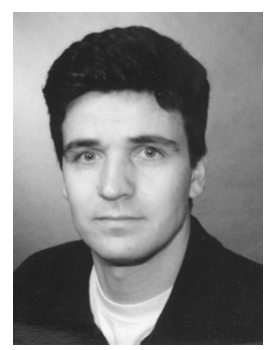

Bernhard Jakoby (S'91-A'95-SM'98) was born in Neuss, Germany, in 1966. He obtained the Dipl.-Ing. (M.Sc.) degree in Communication Engineering and the Ph.D. degree in Electrical Engineering from the Vienna University of Technology, Austria, in 1991 and 1994, respectively.

From 1991 to 1994 he worked as Research Assistant at the Institute of General Electrical Engineering and Electronics at the Vienna University of Technology, Austria. Subsequently, he obtained an Erwin Schrödinger grant from the Austrian Fund for Scientific Research (FWF) to perform research on the electrodynamics of complex media at the Department of Information Technology at the University of Ghent, Belgium. From 1996 to 1999 he held the position of a research associate and later assistant professor at the Electronic Instrumentation Laboratory of the Delft University of Technology, The Netherlands, working on the development of microacoustic sensors. Since 1999, he has been with the Robert Bosch GmbH, Reutlingen, Germany, performing research and development on sensors for automotive applications.

Bernhard Jakoby is a senior member of the IEEE. His research interests are focused on theory and applications in the fields of electromagnetics, microacoustics and integrated sensors.

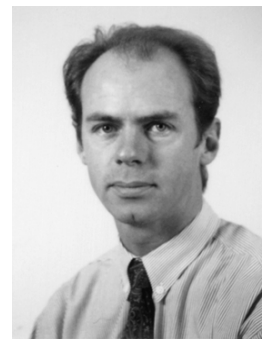

Michael J. Vellekoop was born in Amsterdam in 1960. He received the BSc degree in Physics from the HTS Dordrecht, The Netherlands, and the PhD degree in Electrical Engineering from the Delft University of Technology, The Netherlands, in 1982 and 1994, respectively.

From 1982 to 1984 , he stayed with the Royal Netherlands Naval College as a reserve officer after which he joined the Electronic Instrumentation Laboratory at the Delft University of Technology to work in the field of acoustic wave sensors. In addition, from 1988 to 1996, he was managing director of Xensor Integration B.V. and was involved in the development and the production of silicon sensors and actuators. Currently, he leads the Physical Chemosensors and Microacoustic Devices Group of the Electronic Instrumentation Laboratory, DIMES, where he holds the position of an associate professor. His recent research activities have been in the areas of microacoustic sensor systems for gas and liquid sensing applications, solid state sensor technology, and physical chemosensors. 